\title{
Band-Gap And Vibration-Transmission Properties of a Periodic Compound Plate in Free Boundary Condition
}

\author{
Zhiwei Guo \\ School of Marine Science and Technology \\ Northwestern Polytechnical University \\ Xi'an, China
}

\author{
Meiping Sheng \\ School of Marine Science and Technology \\ Northwestern Polytechnical University \\ Xi'an, China
}

\begin{abstract}
The flexural-wave band-gap and vibration transmission characteristics of a periodic compound plate with free boundary condition are studied in this paper. The exact solutions of the band-gap frequency and the vibration response are obtained by solving governing equations and Bloch-Floquet periodic boundary equations. The finite-element-method (FEM) validation shows that the theoretical solutions have high accuracy and have excellent agreement with the FEM results. In the pass band, the flexural wave propagates normally without attenuation, while in the band-gap, the wave is attenuated with significant attenuation levels. Compared with the previous plane-wave model, the present model gives more accurate results and can represent the realistic situation of a periodic plate structure. Further study shows that the transmission characteristic of a finite periodic compound plate is dependent on the excitation distribution. The band-gap width of a symmetric or anti-symmetric excitation is broader than that of a general excitation. Therefore, when the periodic plate is under symmetric or anti-symmetric load, the attenuation performance of vibration can be improved.
\end{abstract}

Keywords- Periodic compound plate; band-gap; vibration suppression; transfer matrix method

\section{INTRODUCTION}

Periodic structure is composed of a number of identical elements repeated in one, two, and three dimensions [1]. Owing to the Bragg-scattering effect [2] or locally resonant effect [3], the wave filtering phenomenon exists in a periodic structure, with waves in the pass band propagating freely and waves in the band-gap being attenuated gradually [4]. Thus the periodic structure has given a new method to reduce vibration and wave propagation. Attracted by the great potential in vibration and noise control, extensive studies about periodic structures on structure types [5-7], band-gap calculation methods [8-10], and band-gap formation mechanisms [2, 3] have been conducted. In last two decades, phononic crystal [11, 12] and metamaterial [13-15] based on periodic theory have renewed our sight and injected new life to periodic structure.

As plate-type structure is widely used in the engineering applications, the spatial periodicity was introduced in the plate-type structure to reduce noise and vibration in extensive studies, including periodically supported plate [16], periodically stiffened plate [17], a plate with periodically attached spring-mass resonators [18], and a plate with periodically filled-in scattering units $[19,20]$. In addition, there were also a number of works related to periodic plate in various points of views [21-24]. In the previous works about periodic plate, only a couple of studies were associated with periodic compound plate, where two different sub-plates repeat periodically along one direction. Sorokin [25] studied the band-gap performance of a periodic compound plate However, in Sorokin's model, only plane wave was considered and the effects of non-plane wave modes were neglected. Thus, the model was quite like a periodic compound beam, while the effects of Poisson's ratio were considered. In fact, the non-plane wave modes have great effect on the band-gap characteristic. Therefore, the previous model cannot predict the real band-gap characteristic of a periodic compound plate when the plane wave and non-plane wave are coupled together.

In this paper, Sorokin's work is extended, with both the plane wave modes and the non-plane wave modes being included in the present model, resulting that the onedimensional model in Sorokin's work becomes a special case in the present model. By considering all the modes, the present model gives more accurate prediction for the band-gap and transmission characteristic of a periodic compound plate. In addition, the effect of excitation distribution on transmission characteristic of a finite periodic plate is also studied. Symmetric excitation and anti-symmetric excitation are respectively associated with symmetric band-gap and antisymmetric band-gap, which are constituted by symmetric propagation modes and anti-symmetric propagation modes, respectively. Although free boundary condition is considered in this paper, the present model can be easily applied to the clamped, simply supported, or even elastic boundary conditions by simply changing the boundary equations.

\section{THEORETICAL MODEL}

\section{A. Vibration Transmission}

A finite periodic compound plate is shown in Fig. 1, which consists of alternating sub-plates repeated in $x$-direction. A unit element is composed of cell A and cell B, with Young's moduli $E_{\mathrm{A}}$ and $E_{\mathrm{B}}$, densities $\rho_{\mathrm{A}}$ and $\rho_{\mathrm{B}}$, Poisson ratios $v_{\mathrm{A}}$ and $v_{\mathrm{B}}$, lengths $a_{\mathrm{A}}$ and $a_{\mathrm{B}}$, thicknesses $h_{\mathrm{A}}$ and $h_{\mathrm{B}}$, and widths $b_{\mathrm{A}}$ and $b_{\mathrm{B}}\left(b_{\mathrm{A}}=b_{\mathrm{B}}\right)$. The lattice constant is expressed as $a=a_{\mathrm{A}}+a_{\mathrm{B}}$. The forced response is obtained in this subsection to study the vibration transmission under a harmonic force excitaion $f_{0} e^{j \omega t}$ applied at the first cell (cell 0). 


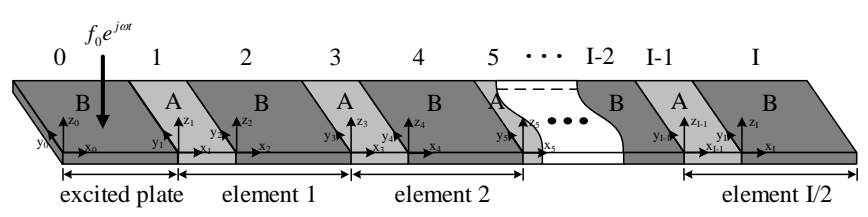

Fig. 1. Schematics of a finite periodic compound plate

The harmonic governing equation of the $i^{\text {th }}$ thin plate is

$$
D_{i} \nabla^{4} w_{i}\left(x_{i}, y_{i}\right)-\rho_{i} h_{i} \omega^{2} w_{i}\left(x_{i}, y_{i}\right)=f_{i},
$$

with bending moment $D_{i}=E_{i} h_{i}^{3} / 12\left(1-v_{i}^{2}\right)$ and $\nabla^{4}=\partial^{4} / \partial x_{i}^{4}+2 \partial^{4} / \partial x_{i}^{2} \partial y_{i}^{2}+\partial^{4} / \partial y_{i}^{4}$. The harmonic solution of (1) can be expressed as [26]

$$
w_{i}\left(x_{i}, \mathrm{y}_{i}\right)=\sum_{m=0}^{M} \sum_{n=0}^{N} A_{i m n} \cos \lambda_{i m} x_{i} \cos \lambda_{i n} y_{i}+p\left(x_{i}, y_{i}\right) \text {, }
$$

where $p\left(x_{i}, y_{i}\right)=\sum_{l=1}^{4}\left[\xi_{i b}^{l}\left(y_{i}\right) \sum_{m=0}^{M} c_{i m}^{l} \cos \lambda_{i m} x_{i}+\xi_{i a}^{l}\left(x_{i}\right) \sum_{n=0}^{N} d_{i n}^{l} \cos \lambda_{i n} y_{i}\right]$, $\lambda_{i m}=m \pi / a_{i}$, and $\lambda_{i n}=n \pi / b_{i}$. The terms $A_{i m n}, c_{i m}^{l}$, and $d_{i n}^{l}$ are unknown coefficients and the terms $\xi_{i a}^{l}\left(x_{i}\right)$ and $\xi_{i b}^{l}\left(y_{i}\right)$ can be obtained from the expression of $\xi_{i s}\left(\tau_{i}\right)(s=a, \tau=x$ or $s=b, \tau=y$ )

$$
\xi_{i s}\left(\tau_{i}\right)=\left[\begin{array}{llll}
\xi_{i s}^{1}\left(\tau_{i}\right) & \xi_{i s}^{2}\left(\tau_{i}\right) & \xi_{i s}^{3}\left(\tau_{i}\right) & \xi_{i s}^{4}\left(\tau_{i}\right)
\end{array}\right]^{\mathrm{T}},
$$

where $\xi_{i s}^{1}\left(\tau_{i}\right)=\sin \left(\pi \tau_{i} / 2 s_{i}\right) \quad, \quad \xi_{i s}^{2}\left(\tau_{i}\right)=\cos \left(\pi \tau_{i} / 2 s_{i}\right)$, $\xi_{i s}^{3}\left(\tau_{i}\right)=\sin \left(3 \pi \tau_{i} / 2 s_{i}\right)$, and $\xi_{i s}^{4}\left(\tau_{i}\right)=\cos \left(3 \pi \tau_{i} / 2 s_{i}\right) . \xi_{i s}\left(\tau_{i}\right)$ can be expanded to series as

$$
\xi_{i s}\left(\tau_{i}\right)=\sum_{r=0}^{\infty} \gamma_{r} \cos \lambda_{i s r} \tau_{i}
$$

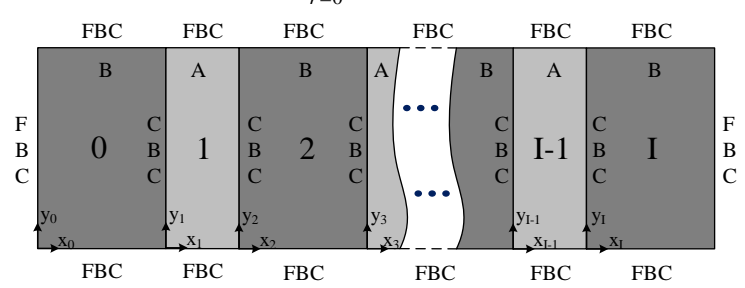

Fig. 2. Boundary conditions of a finite periodic plate

The boundary conditions of a finite periodic plate are shown in Fig. 2, including free (FBC) and continuous (CBC) boundary conditions. The boundary equations are expressed respectively as:

(1) FBCs along $x$-direction $\left(y_{i}=0\right.$ and $y_{i}=b_{i}$ )

$$
\left\{\begin{array}{l}
\left.M_{i y}\right|_{y_{i}=0}=0,\left.\quad Q_{i y}\right|_{y_{i}=0}=0, \\
\left.M_{i y}\right|_{y_{i}=b_{i}}=0,\left.\quad Q_{i y}\right|_{y_{i}=b_{i}}=0
\end{array} \quad(i=0,1, \cdots, \mathrm{I})\right.
$$

(2) FBCs along $y$-direction $\left(x_{0}=0\right.$ and $x_{\mathrm{I}}=a_{\mathrm{I}}$ )

$$
\left.M_{0 x}\right|_{x_{0}=0}=0,\left.Q_{0 x}\right|_{x_{0}=0}=0,\left.M_{\mathrm{I} x}\right|_{x_{\mathrm{I}}=a_{\mathrm{I}}}=0,\left.Q_{\mathrm{I} x}\right|_{x_{\mathrm{I}}=a_{\mathrm{I}}}=0
$$

(3) CBCs between the $(i-1)^{\text {th }}$ cell and the $i^{\text {th }}$ cell $\left(x_{i-1}=a_{i-1}\right.$ or $\left.x_{i}=0\right)$

$$
\left\{\begin{array}{l}
\left.w_{i-1}\right|_{x_{i-1}=a_{i-1}}=\left.w_{i}\right|_{x_{i}=0},\left.\quad \frac{\partial w_{i-1}}{\partial x_{i-1}}\right|_{x_{i-1}=a_{i-1}}=\left.\frac{\partial w_{i}}{\partial x_{i}}\right|_{x_{i}=0}, \quad\left(\begin{array}{l}
i=1,2 \\
, \cdots, \mathrm{I}
\end{array}\right) \\
\left.M_{(i-1) x}\right|_{x_{i-1}=a_{i-1}}=\left.M_{(i) x}\right|_{x_{i}=0},\left.Q_{(i-1) x}\right|_{x_{(i-1)}=a_{(i-1)}}=\left.Q_{(i) x}\right|_{x_{i}=0}
\end{array}\right.
$$

where the bending moments and the shearing forces in the above boundary conditions can be expressed respectively as

$$
\begin{cases}M_{i x}=-D_{i}\left(\frac{\partial^{2} w_{i}}{\partial x_{i}^{2}}+v_{i} \frac{\partial^{2} w_{i}}{\partial y_{i}^{2}}\right), & Q_{i x}=-D_{i}\left[\frac{\partial^{3} w_{i}}{\partial x_{i}^{3}}+\left(2-v_{i}\right) \frac{\partial^{3} w_{i}}{\partial x_{i} \partial y_{i}^{2}}\right], \\ M_{i y}=-D_{i}\left(\frac{\partial^{2} w_{i}}{\partial y_{i}^{2}}+v_{i} \frac{\partial^{2} w_{i}}{\partial x_{i}^{2}}\right), & Q_{i y}=-D_{i}\left[\frac{\partial^{3} w_{i}}{\partial y_{i}^{3}}+\left(2-v_{i}\right) \frac{\partial^{3} w_{i}}{\partial x_{i}^{2} \partial y_{i}}\right]\end{cases}
$$

By substituting $(2-4,8)$ into $(5-7)$, the boundary equations can be obtained in a matrix form

$$
\mathbf{H p}=\mathbf{Q} \mathbf{a} \text {, }
$$

where $\mathbf{p}=\left[\begin{array}{llll}\mathbf{p}_{0} & \mathbf{p}_{1} & \cdots & \mathbf{p}_{\mathrm{I}}\end{array}\right]^{\mathrm{T}}$ and $\mathbf{a}=\left[\begin{array}{llll}\mathbf{a}_{0} & \mathbf{a}_{1} & \cdots & \mathbf{a}_{\mathrm{I}}\end{array}\right]^{\mathrm{T}}$. The terms $\mathbf{p}_{i}$ and $\mathbf{a}_{i}(i=0,1, \cdots, \mathrm{I})$ are defined as $\mathbf{p}_{i}=\left[c_{i 0}^{1}, c_{i 1}^{1}\right.$, $\cdots, c_{i M}^{1}, c_{i 0}^{2}, \cdots, c_{i M}^{2}, c_{i 0}^{3}, \cdots, c_{i M}^{3}, c_{i 0}^{4}, \cdots, c_{i M}^{4}, d_{i 0}^{1}, d_{i 1}^{1}, \cdots$, $\left.d_{i N}^{1}, d_{i 0}^{2}, \cdots, d_{i N}^{2}, d_{i 0}^{3}, \cdots, d_{i N}^{3}, d_{i 0}^{4}, \cdots, d_{i N}^{4}\right]$ and $\mathbf{a}_{i}=\left[A_{i 00}\right.$, $A_{i 01}, \cdots, A_{i 0 N}, A_{i 10}, A_{i 11}, \cdots, A_{i 1 N}, \cdots, A_{i m n}, \cdots, A_{i M 0}, A_{i M 1}$, $\left.A_{i M N}\right]$, respectively.

Thus from the boundary equation, $\mathbf{p}$ is expressed by $\mathbf{a}$ as $\mathbf{p}=\mathbf{H}^{-1} \mathbf{Q} \mathbf{a}$. For the governing equations, the excitation force $f_{i}$ in (1) is expressed by modal forces in series form

$D_{i} \nabla^{4} w_{i}\left(x_{i}, y_{i}\right)-\rho_{i} h_{i} \omega^{2} w_{i}\left(x_{i}, y_{i}\right)=\sum_{m=0}^{M} \sum_{n=0}^{N} F_{i m n} \cos \lambda_{i m} x_{i} \cos \lambda_{i n} y_{i}$.

Substituting (2-4) into (10) gives

$$
\mathbf{K}_{1} \mathbf{a}+\mathbf{S p}-\omega^{2}\left(\mathbf{M}_{1} \mathbf{a}+\mathbf{T p}\right)=\mathbf{F}
$$

where $\quad \mathbf{F}_{i}=\left[F_{i 00}, F_{i 01}, \cdots, F_{i 0 N}, F_{i 10}, \cdots, F_{i 1 N}, \cdots, F_{i M 0}, \cdots, F_{i M N}\right]$ and $\mathbf{F}=\left[\begin{array}{llll}\mathbf{F}_{0} & \mathbf{F}_{1} & \cdots & \mathbf{F}_{\mathrm{I}}\end{array}\right]^{\mathrm{T}}$. Substituting the formulation $\mathbf{p}=\mathbf{H}^{-1} \mathbf{Q} \mathbf{a}$ into (11) gives

$$
\left[\mathbf{K}-\omega^{2} \mathbf{M}\right] \mathbf{a}=\mathbf{F}
$$

where $\mathbf{K}=\mathbf{K}_{1}+\mathbf{S} \mathbf{H}^{-1} \mathbf{Q}$ and $\mathbf{M}=\mathbf{M}_{1}+\mathbf{T H} \mathbf{H}^{-1} \mathbf{Q}$.

At a given frequency, the eigenvector a can be obtained by solving (12), and then $\mathbf{p}$ is also determined by (9). As a and $\mathbf{p}$ become known, the vibration response of each cell will be finally determined.

\section{B. Band-gap Formulation}

The band-gap frequency can be determined by the analysis of a unit element with Bloch-Floquet periodic condition. As shown in Fig. 3, a unit element consists of three types of boundary conditions, including free (FBC), continuous (CBC), and periodic $(\mathrm{PBC})$ boundary conditions. 


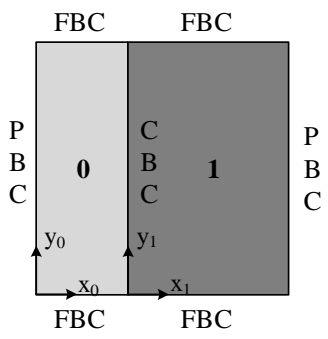

Fig. 3. Boundary conditions of a unit element

The boundary equations of $\mathrm{FBC}$ and $\mathrm{CBC}$ have been shown in (5) and (7), while the boundary equations of PBC can be expressed as

$$
\left\{\begin{array}{ll}
\left.w_{0}\right|_{x_{0}=0}=\left.e^{-i q a} w_{1}\right|_{x_{1}=a_{1}}, & \left.\frac{\partial w_{0}}{\partial x_{0}}\right|_{x_{0}=0}=\left.e^{-i q a} \frac{\partial w_{1}}{\partial x_{1}}\right|_{x_{1}=a_{1}}, \\
\left.M_{0 x}\right|_{x_{0}=0}=\left.e^{-i q a} M_{1 x}\right|_{x_{1}=a_{1}}, & \left.Q_{0 x}\right|_{x_{0}=0}=\left.e^{-i q a} Q_{1 x}\right|_{x_{1}=a_{1}}
\end{array},\right.
$$

where $q$ is wavenumber, which represents the wave propagation and wave attenuation performance. Equation (13) can be rewritten as a matrix form

$$
\overline{\mathbf{H}}(q) \overline{\mathbf{p}}=\overline{\mathbf{Q}}(q) \overline{\mathbf{a}},
$$

where $\overline{\mathbf{a}}=\left[\begin{array}{ll}\mathbf{a}_{0} & \mathbf{a}_{1}\end{array}\right]^{\mathrm{T}}$ and $\overline{\mathbf{p}}=\left[\begin{array}{ll}\mathbf{p}_{0} & \mathbf{p}_{1}\end{array}\right]^{\mathrm{T}}$. From (14), $\overline{\mathbf{p}}$ can be expressed as $\overline{\mathbf{p}}=\overline{\mathbf{H}}(q)^{-1} \overline{\mathbf{Q}}(q) \overline{\mathbf{a}}$. By substituting (2-4) into the homogeneous plate equation (set $f_{i}=0$ in (1)), it is obtained that

$$
\overline{\mathbf{K}}_{1} \overline{\mathbf{a}}+\overline{\mathbf{S}} \overline{\mathbf{p}}-\omega^{2}\left(\overline{\mathbf{M}}_{1} \overline{\mathbf{a}}+\overline{\mathbf{T}} \overline{\mathbf{p}}\right)=\mathbf{0} .
$$

Substituting $\overline{\mathbf{p}}=\overline{\mathbf{H}}(q)^{-1} \overline{\mathbf{Q}}(q) \overline{\mathbf{a}}$ into (15) gives

$$
\left[\overline{\mathbf{K}}(q)-\omega^{2} \overline{\mathbf{M}}(q)\right] \overline{\mathbf{a}}=\mathbf{0}
$$

where $\overline{\mathbf{K}}=\overline{\mathbf{K}}_{1}+\overline{\mathbf{S}} \overline{\mathbf{H}}(q)^{-1} \overline{\mathbf{Q}}(q)$ and $\overline{\mathbf{M}}=\overline{\mathbf{M}}_{1}+\overline{\mathbf{T}} \overline{\mathbf{H}}(q)^{-1} \overline{\mathbf{Q}}(q)$.

When the wavenumber $q$ is given, the stiffness matrix $\overline{\mathbf{K}}$ and mass matrix $\overline{\mathbf{M}}$ become known. The normalized wavenumber is defined as $q_{n}=q a / \pi$. Sweeping $q_{n}$ in one period from -1 to 1 will give rise to the dispersion curves (the frequency $\omega$ as a function of $q_{n}$ ), and then the band-gap properties of the periodic compound plate can be finally determined.

\section{BAND-GAP CHARACTERISTIC AND VALIDATION}

The band-gap characteristic of an infinite periodic plate is studied in this section. The plate parameters are given as $E_{\mathrm{A}}=2 \mathrm{GPa} \quad, \quad E_{\mathrm{B}}=210 \mathrm{GPa} \quad, \quad \rho_{\mathrm{A}}=1142 \mathrm{~kg} / \mathrm{m}^{3}$ $\rho_{\mathrm{B}}=7800 \mathrm{~kg} / \mathrm{m}^{3}, v_{\mathrm{A}}=0.39, v_{\mathrm{B}}=0.30, a_{\mathrm{A}}=0.15 \mathrm{~m}$, $a_{\mathrm{B}}=0.35 \mathrm{~m}, b_{\mathrm{A}}=b_{\mathrm{B}}=0.5 \mathrm{~m}$, and $h_{\mathrm{A}}=h_{\mathrm{B}}=5 \mathrm{~mm}$. The dispersion curves below $400 \mathrm{~Hz}$ are shown in Fig. 4. As we can see in the figure, the dispersion curves of a periodic compound plate are constituted by several dispersion branches, with each branch corresponding to a specific wavepropagation mode (see Fig. 5). These branches are divided into several modal groups according to their cross-stream modal shapes. As shown in Fig. 4 and Fig. 5, the dispersion branches below $400 \mathrm{~Hz}$ are divided into four groups, the $1^{\text {st }}$ mode group $\left(1^{1 \#}, 1^{2 \#}, 1^{3 \#}\right.$, and $\left.1^{4 \#}\right)$, the $2^{\text {nd }}$-mode group $\left(2^{1 \#}\right.$, $2^{2 \#}$, and $\left.2^{3 \#}\right)$, the $3^{\text {rd }}$-mode group $\left(3^{1 \#}, 3^{2 \#}\right.$, and $\left.3^{3 \#}\right)$, and the $4^{\text {th }}$ mode group $\left(4^{1 \#}\right.$ and $\left.4^{2 \#}\right)$.

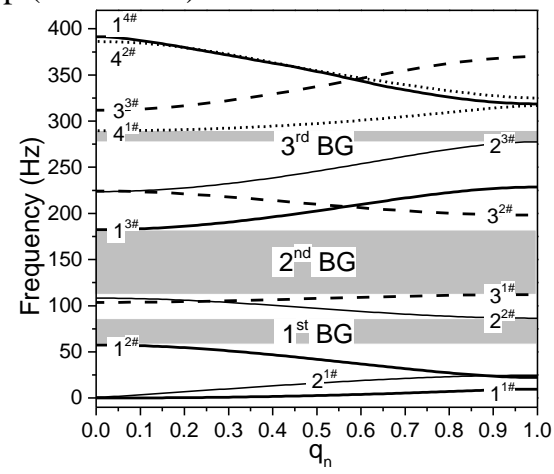

Fig. 4. Dispersion curves of the $1^{\text {st }}$-mode group ('-'), $2^{\text {nd }}$-mode group (''), $3^{\text {rd }}$-mode group ('---'), and $4^{\text {th }}$-mode group (' $\ldots . .$. ') (BG: band-gap)

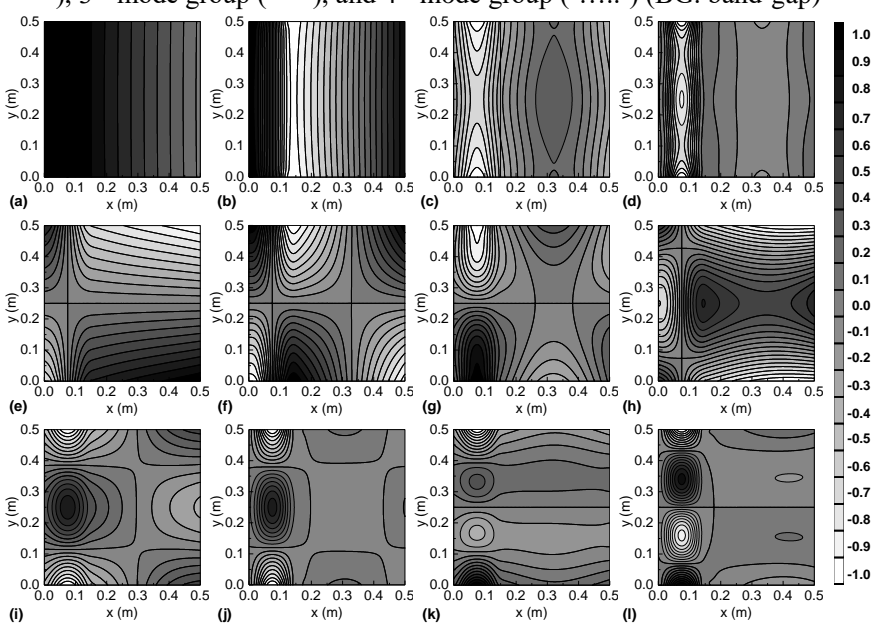

Fig. 5. Modal shapes of a periodic compound plate at $q_{\mathrm{n}}=0.25$ for the $1^{\text {st }}$ mode group ((a) $1^{1 \#}$, (b) $1^{2 \#}$, (c) $1^{3 \#}$, and (d) $1^{4 \#)}$ ), $2^{\text {nd }}$-mode group (I $2^{1 \#}$, (f) $2^{2 \#}$, and $\left.(\mathrm{g}) 2^{3 *}\right), 3^{\text {rd }}$-mode group ((h) $3^{1 \#}$, (i) $3^{2 \#}$, and (j) $\left.3^{3 \#}\right)$, and $4^{\text {th }}$-mode group ((k) $4^{1 \#}$ and (l) $\left.4^{2 \#}\right)$

The dispersion branches from the same modal group are separated between each other, and the frequency gap between neighboring dispersion branches constitute the modal bandgap. For each modal band-gap, the wave cannot propagate with its corresponding modal shape, while it may propagate with the modal shapes belonging to other modal groups. For example, the wave of $100 \mathrm{~Hz}$ cannot propagate with the modal shape in the $1^{\text {st }}$-mode group, however, it can propagate with mode $2^{2 \#}$ in the $2^{\text {nd }}$-mode group. For the wave with a specific frequency, if there's no any dispersion curve related to this frequency, the wave cannot propagate with any modal shape. Thus the general band-gap is the intersection of all the modal band-gaps.

As shown in Fig. 4, the band-gap and band-pass alternate with each other. Three band-gaps exist below $400 \mathrm{~Hz}$, namely $57.5 \mathrm{~Hz}-86.5 \mathrm{~Hz}, \quad 112.1 \mathrm{~Hz}-182.3 \mathrm{~Hz}, \quad$ and $277.5 \mathrm{~Hz}-$ $289.5 \mathrm{~Hz}$. The first band-gap is in low frequency range, thus it can be used in the low frequency vibration control. The total band-gap width is $111.2 \mathrm{~Hz}$, with the band-gap ratio exceeding $25 \%$, which indicates that more than a quarter of flexural wave between $0 \mathrm{~Hz}$ and $400 \mathrm{~Hz}$ are suppressed. The dispersion curves are also calculated with FEM by COMSOL- 
Multiphysics software with the results shown in Fig. 6(a). It can be seen that the dispersion curves from present model have an excellent agreement with the results of an FEM model, with no more than $2.5 \mathrm{~Hz}$ difference. Thus, the theoretical model derived in this paper has an excellent accuracy.
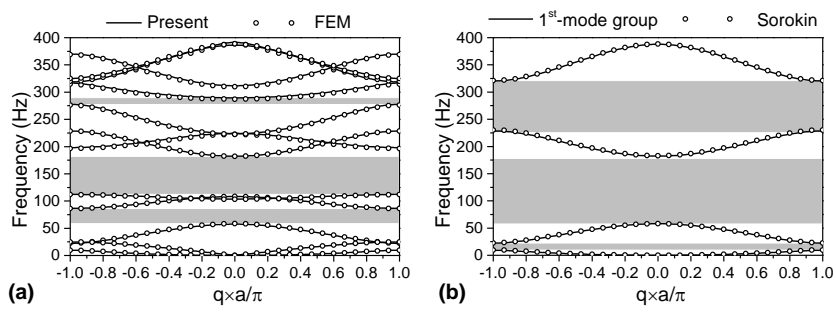

Fig. 6. Dispersion curves and band-gaps for (a) comparisons between present model and FEM model and (b) comparisons between the 1st-mode group in present model and Sorokin model (The shaded region in gray are band-gaps.)

S.V. Sorokin has studied the band-gaps of a periodic compound plate where only plane-wave propagation modes are considered, where the dispersion curves correspond to the dispersion branches of the $1^{\text {st }}$-mode group in Fig. 4 . The two results are compared in Fig. 6(b) showing perfect coincidence. As shown in Fig. 6, the dispersion curves of present model (Fig. 6(a)) are more complicated than those of plane wave model (Fig. 6(b)), and the plane-wave model in Sorokin's work has become a special case of this general plate model. As the non-plane wave modes are included in present model, the band-gap width become narrower. It can observed in Fig. 6 that the first band-gap $(9.1 \mathrm{~Hz}-22.4 \mathrm{~Hz})$ of the planewave model disappears in the present model; the second bandgap $(58.1 \mathrm{~Hz}-182.9 \mathrm{~Hz})$ of the plane-wave model is divided into two narrow band-gaps $(57.5 \mathrm{~Hz}-86.5 \mathrm{~Hz}$ and $112.1 \mathrm{~Hz}-$ $182.3 \mathrm{~Hz}$ ) in the present model; the third band-gap $(230.2 \mathrm{~Hz}-321.0 \mathrm{~Hz})$ of the plane-wave model is changed to a smaller band-gap $(277.5 \mathrm{~Hz}-289.5 \mathrm{~Hz})$ in the present model. Thus, when the non-plane-wave propagation modes are considered, the band-gap performance varies significantly and the band-gap properties predicted by the plane-wave model cannot represent the realistic wave propagation situations, while the present model will provide good results.

\section{VIBRATION TRANSMISSION}

The vibration transmission characteristic of a finite periodic compound plate (see Fig. 1) is studied in this section with the same material and geometry parameters shown in section 3. A harmonic point force is applied at $x_{0}=a_{0} / 4$ and $y_{0}=b_{0} / 4$ in the first plate (cell 0 ). The vibration transmittance is defined as $T_{e}=20 \log 10\left(\bar{v}_{\mathrm{I}} / \bar{v}_{0}\right)$, where $\bar{v}_{0}$ and $\bar{v}_{\mathrm{I}}$ are the spatially averaged transverse velocities of cell 0 and cell I, respectively. The vibration transmittances of a periodic compound plate with four elements by both the present model and the FEM model are shown in Fig. 7(a). It is observed that the result from the present model has a good coincidence with that from the FEM model. Therefore, the present vibrational model has high accuracy in calculating the vibration response.
It can be seen in Fig. 7(a) that there are three transmission valleys, where the vibrations are significantly attenuated and only very small vibration energy transmits from the first cell (excited cell) to the last one. The band-gaps in the infinite periodic plate calculated in section 3 are displayed in shaded region in gray. As shown in Fig. 7(a), the transmission valleys in a finite periodic plate match very well with the band-gaps in an infinite periodic plate. Thus, the waves in pass bands can propagate freely along the axial direction, while the waves in band-gaps are attenuated with considerable levels. This filtering phenomenon is beneficial to noise and vibration control.
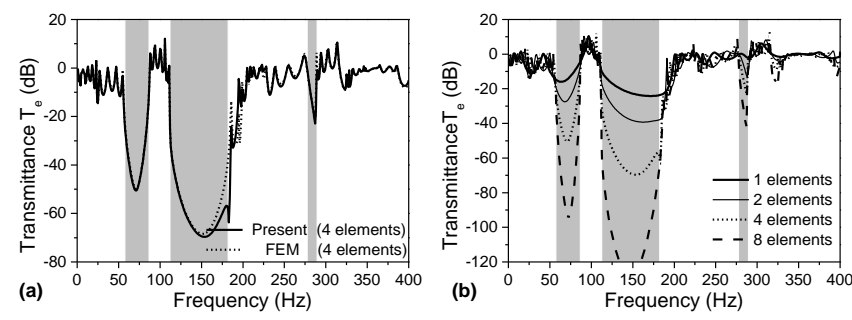

Fig. 7. Vibration transmittance of a periodic compound plate with (a) the comparison between present model and FEM model and (b) the performances with the variation of the number of element (The shaded region in gray are band-gaps.)

The effect of the number of element on transmission characteristic is studied with the number of element equaling one, two, four, and eight and the comparison results are plotted in Fig. 7(b). Significant attenuation levels can be observed for the first and second band-gaps. Even with one element, the averaged attenuation level can reach to about $12 \mathrm{~dB}$ for the first band-gap and $20 \mathrm{~dB}$ for the second bandgap. The attenuation ability of the third band-gap is not as strong as the first two and there's a very little attenuation with one element. With the increase of the number of element, the attenuation levels in the band-gaps increase significantly. When the number of element increases from one to eight, the average attenuation levels increase from $12.7 \mathrm{~dB}$ to $71.5 \mathrm{~dB}$ for the first band-gap, from $20.8 \mathrm{~dB}$ to $107.9 \mathrm{~dB}$ for the second band-gap, and from $0.7 \mathrm{~dB}$ to $25.0 \mathrm{~dB}$ for the third band-gap. The attenuation ability of the third band-gap is weaker than that of the first two band-gaps, hence more elements are needed to achieve a good vibrational performance for the third band-gap.

\section{EFFECT OF EXCITATION DISTRIBUTION}

In this section, the effect of excitation distribution on band-gap property is studied, including point and line excitations with symmetric and anti-symmetric distributions on the plate. Further examination shows that the excitation distribution has great effect on the wave transmission characteristic of a finite periodic compound plate, with significant difference between a symmetric excitation and an anti-symmetric excitation. The band-gaps calculated in section 3 for the infinite periodic plate cannot predict the transmission valleys in a finite periodic plate when the excitation is symmetrically or anti-symmetrically distributed. In fact, a symmetric or anti-symmetric excitation always causes better performance than a general excitation. This phenomenon can be explained by examining corresponding dispersion curves and propagation modes. 
It is observed in Fig. 5 that the modal shapes are either symmetrically distributed (the $1^{\text {st }}$-mode and $3^{\text {rd }}$-mode groups) or anti-symmetrically distributed (the $2^{\text {nd }}$-mode and $4^{\text {th }}$-mode groups). As shown in Fig. 8, the symmetric band-gaps are constituted by the symmetric dispersion branches $\left(1^{1 \#}, 1^{2 \#}, 1^{3 \#}\right.$, $1^{4 \#}, 3^{1 \#}, 3^{2 \#}$, and $\left.3^{3 \#}\right)$ and the anti-symmetric band-gaps are associated with the anti-symmetric dispersion branches $\left(2^{1 \#}\right.$, $2^{2 \#}, 2^{3 \#}, 4^{1 \#}$, and $\left.4^{2 \#}\right)$. The band-gap frequencies are listed in Table 1. By comparing Fig. 4, Fig. 8, and Table 1, it is found that the general band-gaps are the intersection of symmetric band-gaps and anti-symmetric band-gaps. For example, the first general band-gap $(57.5 \mathrm{~Hz}-86.5 \mathrm{~Hz})$ is the intersection of the second symmetric band-gap $(57.5 \mathrm{~Hz}-103.7 \mathrm{~Hz})$ and the first anti-symmetric band-gap $(24.7 \mathrm{~Hz}-86.5 \mathrm{~Hz})$. The total band-gap widths of the symmetric and anti-symmetric band-gaps are respectively $217.1 \mathrm{~Hz}$ and $202.4 \mathrm{~Hz}$, which are broader than that of the general band-gap width with $111.2 \mathrm{~Hz}$. Thus, when all of the waves are propagating in symmetric or anti-symmetric modes, the periodic compound plate will have a better performance in the aspect of band-gap width.
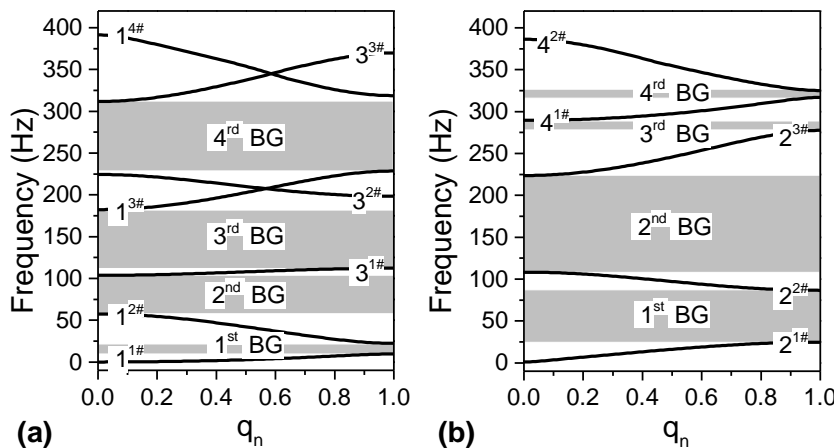

Fig. 8. Dispersion curves and band-gaps of (a) symmetric modes and (b) anti-symmetric modes (BG: band-gap)

TABLE I. COMPARISON OF THE BAND-GAPS

\begin{tabular}{|c|c|c|c|}
\hline $\begin{array}{c}\text { Band-gap } \\
\text { order }\end{array}$ & $\begin{array}{c}\text { Symmetric } \\
\text { band-gaps }\end{array}$ & $\begin{array}{c}\text { Anti-Symmetric } \\
\text { band-gaps }\end{array}$ & $\begin{array}{c}\text { General band- } \\
\text { gaps }\end{array}$ \\
\hline First & $9.6-22.2 \mathrm{~Hz}$ & $24.7-86.5 \mathrm{~Hz}$ & $57.5-86.5 \mathrm{~Hz}$ \\
\hline Second & $57.5-103.7 \mathrm{~Hz}$ & $108.2-223.6 \mathrm{~Hz}$ & $112.1-182.3 \mathrm{~Hz}$ \\
\hline Third & $112.1-182.3 \mathrm{~Hz}$ & $277.5-289.5 \mathrm{~Hz}$ & $277.5-289.5 \mathrm{~Hz}$ \\
\hline Fourth & $228.5-311.7 \mathrm{~Hz}$ & $316.8-325.1 \mathrm{~Hz}$ & $\mathrm{~N} / \mathrm{A}$ \\
\hline Five & $342.4-347.3 \mathrm{~Hz}$ & $\mathrm{~N} / \mathrm{A}$ & $\mathrm{N} / \mathrm{A}$ \\
\hline
\end{tabular}

The vibration transmittances of a finite periodic compound plate (see Fig. 1) with four elements are examined with different force excitations. The positions of point and line excitations are shown in Fig. 9. Four cases of point excitation are examined with case 1: excitation at $\mathrm{p}_{1}$ with $F_{1}=1 \mathrm{~N}$, case 2: excitation at position $\mathrm{p}_{2}$ with $F_{2}=1 \mathrm{~N}$, case 3 : symmetric excitation at $\mathrm{p}_{1}$ and $\mathrm{p}_{3}$ with $F_{1}=1 \mathrm{~N}$ and $F_{3}=1 \mathrm{~N}$, and case 4 : anti-symmetric excitation at $\mathrm{p}_{1}$ and $\mathrm{p}_{3}$ with $F_{1}=1 \mathrm{~N}$ and $F_{3}=-1 \mathrm{~N}$. The vibration transmittances are shown in Fig. 10. It can be seen that the transmittance characteristics of the finite periodic compound plate are dependent on excitation types. For a symmetric force excitation (see Fig. 10(b, c)), the transmittance valleys are in coincidence with the symmetric band-gaps. On the contrary, for the anti-symmetric force excitation (see Fig. 10(d)), the transmittance valleys are in coincidence with the anti-symmetric band-gaps. However when the force excitation is neither symmetric nor antisymmetric, the transmittance valleys are in coincidence with the general band-gaps.
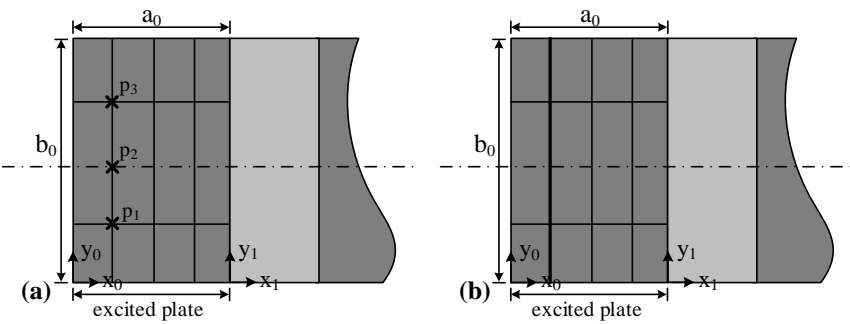

Fig. 9. Schematics of (a) point excitation at $\mathrm{p}_{1}\left(a_{0} / 4, b_{0} / 4\right), \mathrm{p}_{2}\left(a_{0} / 4, b_{0} / 2\right)$, and $\left.\mathrm{p}_{3}\left(a_{0} / 4,3 b_{0} / 4\right)\right)$ and (b) line excitation at $\left.x_{0}=a_{0} / 4\right)$
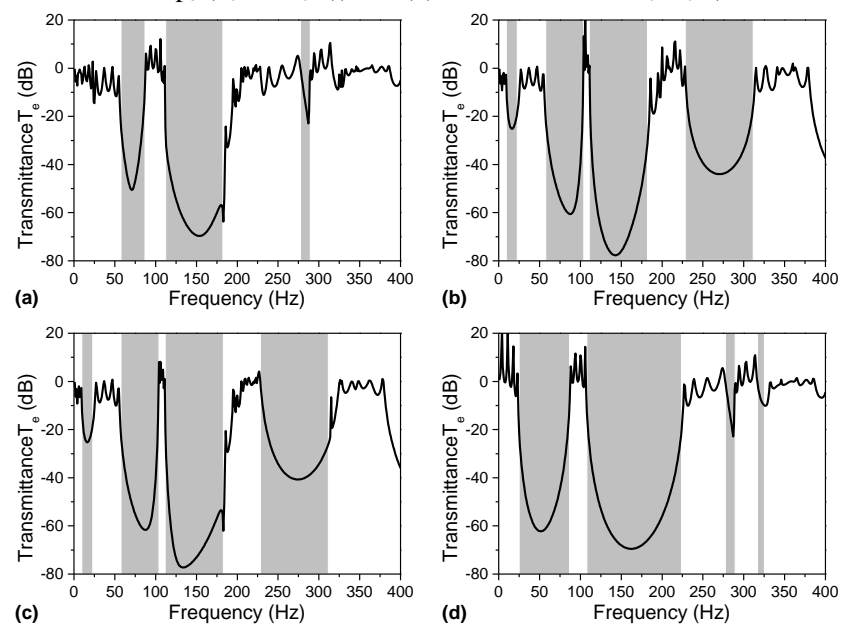

Fig. 10. Vibration transmittances under point excitations of (a) case 1, (b) case 2 , (c) case 3 , and (d) case 4

Five more cases of line excitation applied at $x_{0}=a_{0} / 4$ are also studied with case 5: $F\left(y_{0}\right)=1$, case 6: $F\left(y_{0}\right)=\cos \left(\lambda_{2} y_{0}\right)$, case 7: $F\left(y_{0}\right)=\sin \left(\lambda_{1} y_{0}\right)$, case 8: $F\left(y_{0}\right)=\cos \left(\lambda_{1} y_{0}\right)$, and case 9: $F\left(y_{0}\right)=\sin \left(\lambda_{2} y_{0}\right)$, where $\lambda_{1}=\pi / b_{0}$ and $\lambda_{2}=2 \pi / b_{0}$. The force distribution shapes are shown in Fig. 11 and the corresponding vibration transmittances are shown in Fig. 12. The vibration transmittances of line excitations are similar to those of the point excitations, with symmetric line excitations associated with symmetric band-gaps (see Fig. 12(a)) and anti-symmetric line excitations associated with anti-symmetric band-gaps (see Fig. 12(b)).

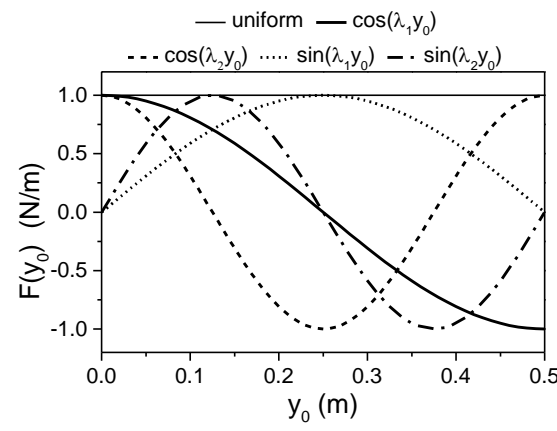

Fig. 11. Force distribution shapes of line excitations along $y$-direction 

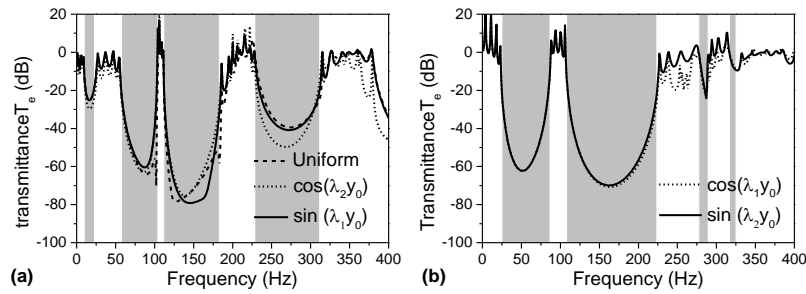

Fig. 12. Vibration transmittances under line excitations of (a) symmetric distribution (case 5, case 6, and case 7) and (b) anti-symmetric distribution (case 8 and case 9)

Therefore, for both point and line force excitations, the excitation distributions have significant effect on wave transmission. The vibration transmittance valleys of symmetric excitation can be predicted by the symmetric bandgaps and those of the anti-symmetric excitation can be predicted by the anti-symmetric band-gaps. When the periodic plate is excited by a symmetric force, half of an antisymmetric mode makes positive contribution to the response and the other half makes equaling negative contribution, resulting that the total contribution from an anti-symmetric mode is zero. However, both of the two half symmetric modes make positive contribution, causing that the total contribution from a symmetric mode is positive. It's the same reason that when the periodic plate is excited by an anti-symmetric force, and only anti-symmetric mode makes positive contribution to the vibration response. Therefore, the symmetric force is associated with the symmetric modes and the anti-symmetric force is associated with the anti-symmetric modes. When the periodic plate is excited by neither symmetric nor antisymmetric force, both the symmetric and anti-symmetric modes will make contribution to the vibration response, and the transmittance valleys can be predicted by the general band-gaps.

From the above, the vibration suppression performance of a finite periodic compound plate is dependent on the force distributions. The symmetric or anti-symmetric force causes better performance than a general force, because the bandwidths of the symmetry band-gap and the anti-symmetry band-gap are broader than that of the general band-gap. Thus, when the periodic plate is used in the practical application, making the engine or excitation set work at a symmetric or anti-symmetric excitation situation, the vibration suppression performance of the periodic plate can be improved significantly. Therefore, in order to reduce more noise and vibration, the excitation set should be installed at the middle line, and also, if there are two identical excitation sets, symmetric installment will be a good choice to reduce noise and vibration.

\section{CONCLUSIONS}

The flexural-wave band-gap characteristic of an infinite periodic compound plate and the transmission characteristic of a finite periodic compound plate are examined in this paper. The exact solutions of band-gap frequency and vibration response are obtained by theoretical derivation and are validated by FEM model. The vibration can be significantly reduced in the band-gaps. Compared with the previous planewave model, the present model gives more accurate results and can represent the realistic situation. The dispersion curves can be divided into two groups, which are associated with symmetric and anti-symmetric modes, resulting that the vibration transmission characteristic of a finite periodic compound plate is dependent on the force distributions. When the force is symmetric, the transmission valleys can be predicted by the symmetric band-gaps; while for the antisymmetric force, the transmission valleys can be predicted by the anti-symmetric band-gaps. A general force, neither symmetric nor anti-symmetric, makes the transmission valleys depending on the general band-gaps, which is narrower in band-gap width than symmetric or anti-symmetric band-gaps. Thus when the force is in symmetric or anti-symmetric situation, the vibration suppression performance of a periodic compound plate will be improved and more vibration can be reduced.

\section{REFERENCES}

[1] Brillouin, L., Wave propagation in periodic structures: Electric filters and crystal lattices, Courier Corporation, 2003.

[2] Sutter-Widmer, D., Deloudi, S. and Steurer, W., Prediction of braggscattering-induced band gaps in phononic quasicrystals, Phys. Rev. B, Vol. 75(9), pp. 094304, 2007.

[3] Liu, Z., Zhang, X., Mao, Y., Zhu, Y. Y., Yang, Z., Chan, C. T. and Sheng, P., Locally resonant sonic materials, Science, Vol. 289(5485), pp. 1734-1736, 2000.

[4] Mead, D. M., Wave propagation in continuous periodic structures: Research contributions from southampton, 1964-1995, J. Sound Vib. Vol. 190(3), pp. 495-524, 1996.

[5] Lee, S., Vlahopoulos, N. and Waas, A. M., Analysis of wave propagation in a thin composite cylinder with periodic axial and ring stiffeners using periodic structure theory, J. Sound Vib., Vol. 329(16) pp. 3304-3318, 2010.

[6] Casadei, F. and Bertoldi, K., Wave propagation in beams with periodic arrays of airfoil-shaped resonating units, J. Sound Vib., Vol. 333(24), pp. 6532-6547, 2014.

[7] Dai, L., Jiang, S., Lian, Z., Hu, H. and Chen, X., Locally resonant band gaps achieved by equal frequency shunting circuits of piezoelectric rings in a periodic circular plate, J. Sound Vib., Vol. 337, pp. 150-160, 2015 .

[8] Saeed, H. M. and Vestroni, F., Simulation of combined systems by periodic structures: The wave transfer matrix approach, J. Sound Vib. Vol. 213(1), pp. 55-74, 1998.

[9] Mead, D. J., The forced vibration of one-dimensional multi-coupled periodic structures: An application to finite element analysis, J. Sound Vib., Vol. 319(1), pp. 282-304, 2009.

[10] Huang, J. and Shi, Z., Attenuation zones of periodic pile barriers and its application in vibration reduction for plane waves, J. Sound Vib., Vol. 332(19), pp. 4423-4439, 2013.

[11] Mohammadi, S., Eftekhar, A. A., Khelif, A., Hunt, W. D. and Adibi A., Evidence of large high frequency complete phononic band gaps in silicon phononic crystal plates, Appl. Phys. Lett., Vol. 92(22), pp. 221905, 2008

[12] Hussein, M. I., Leamy, M. J. and Ruzzene, M., Dynamics of phononic materials and structures: Historical origins, recent progress, and future outlook, Appl. Mech. Rev., Vol. 66(4), pp. 040802, 2014.

[13] Huang, H. H., Sun, C. T. and Huang, G. L., On the negative effective mass density in acoustic metamaterials, Int. J. Eng. Sci., Vol. 47(4), pp. 610-617, 2009.

[14] Pope, S. A. and Daley, S., Viscoelastic locally resonant double negative metamaterials with controllable effective density and elasticity, Phys Lett. A, Vol. 374(41), pp. 4250-4255, 2010.

[15] Del Vescovo, D. and Giorgio, I., Dynamic problems for metamaterials: Review of existing models and ideas for further research, Int. J. Eng. Sci., Vol. 80, pp. 153-172, 2014

[16] Gupta, G. S., Natural flexural waves and the normal modes of periodically-supported beams and plates, J. Sound Vib., Vol. 13(1), pp. 89-101, 1970.

[17] Mace, B. R., Periodically stiffened fluid-loaded plates, i: Response to convected harmonic pressure and free wave propagation, J. Sound Vib., Vol. 73(4), pp. 473-486, 1980 
[18] Xiao, Y., Wen, J. and Wen, X., Flexural wave band gaps in locally resonant thin plates with periodically attached spring-mass resonators Journal of Physics D: Applied Physics, Vol. 45(19), pp. 195401, 2012.

[19] Hsu, J. C. and Wu, T. T., Efficient formulation for band-structure calculations of two-dimensional phononic-crystal plates, Phys. Rev. B Vol. 74(14), pp. 144303, 2006.

[20] Hsu, J. C. and Wu, T. T., Lamb waves in binary locally resonant phononic plates with two-dimensional lattices, Appl. Phys. Lett., Vol. 90(20), pp. 201904, 2007.

[21] Jędrysiak, J., The tolerance averaging model of dynamic stability of thin plates with one-directional periodic structure, Thin Wall. Struct., Vol. 45(10), pp. 855-860, 2007.

[22] Lagarkov, A. N., Kisel, V. N. and Semenenko, V. N., Wide-angle absorption by the use of a metamaterial plate, Prog. Electromagn. Res., Vol. 1, pp. 35-44, 2008.
[23] Casadei, F., Ruzzene, M., Dozio, L. and Cunefare, K. A., Broadband vibration control through periodic arrays of resonant shunts: Experimental investigation on plates, Smart Mater. Struct., Vol. 19(1), pp. 015002, 2009.

[24] Zhu, R., Huang, G. L., Huang, H. H. and Sun, C. T., Experimental and numerical study of guided wave propagation in a thin metamaterial plate, Phys. Lett. A, Vol. 375(30), pp. 2863-2867, 2011.

[25] Sorokin, S. V. and Ershova, O. A., Plane wave propagation and frequency band gaps in periodic plates and cylindrical shells with and without heavy fluid loading, J. Sound Vib., Vol. 278(3), pp. 501-526, 2004.

[26] Li, W. L., Zhang, X., Du, J. and Liu, Z., An exact series solution for the transverse vibration of rectangular plates with general elastic boundary supports, J. Sound Vib., Vol. 321(1), pp. 254-269, 2009. 